\title{
Obstacles in Moving to Agile Software Development Methods; at a Glance
}

\author{
Taghi Javdani Gandomani, Hazura Zulzalil, \\ Abdul Azim Abdul Ghani, Abu Bakar Md. Sultan and Mina Ziaei Nafchi \\ Department of Information Systems, Faculty of Computer Science and Information Technology, \\ University Putra Malaysia (UPM), 43400, Serdang, Malaysia
}

Received 2012-10-03, Revised 2013-04-08; Accepted 2013-05-25

\begin{abstract}
It is only less than a decade that agile SD methods were introduced and got popular steadily. The defined values in these methods and their outcomes have motivated many software producers to use these methods. Since migration from traditional software development methods to agile methods is growing highly, managers of the companies should be aware of problems, hindrances and challenges they may face with during the agile transformation process. This study focused on challenges which companies may face with and it is necessary that managers think about solving them. Classifying them into four main categories; organization and management, people, process and tools are the areas that challenges have been seen in recent studies.
\end{abstract}

Keywords: Agile Software Methods, Agile Software Development, Agile Adoption, Obstacles in Agile Transformation, Agile Transformation Process

\section{INTRODUCTION}

For more than 40 years, traditional Software Development (SD) methods were widely used by all of the software developers. Waterfall model and its incremental methods like Spiral were popular in the entire world. They were supported by huge amount of detailed documentation and valuable experiences. Nevertheless, an innovator group of software experts, by introducing of agility in the SD process, called agile manifesto, suggested changing the mindset of traditional SD formally (Beck et al., 2001; Dingsoyr et al., 2012). However this manifesto did not clearly disagree with traditional SD methods, but, it accentuated values which were against them. In fact achieving agile values was only possible by refusing many activities in traditional SD methods. Traditional SD advocators were cautious in dealing with agile manifesto, but after Boehm's note (Boehm, 2002), which implicitly accepted agile principles and values; a positive atmosphere was gradually created for promoting agile methods.
Although agile production wasn't a new idea in other industries (Sims and Johnson, 2012), but, proposing agility in software industry was completely innovative. Now, after a decade of agile manifesto, many agile methods were introduced. Scrum, Extreme Programming (XP), Lean SD, Crystal, FDD and TDD are some of the agile methods that are used for managing SD process (Cohn, 2009; Dingsoyr et al., 2012). Each of these methods emphasizes on one or more values introduced in agile manifesto and includes many particular activities. Despite of concerns about feasibility of this new approach, customer satisfaction and high quality production (Glazer, 2010) persuade software practitioners to use these methods. Nowadays reputed companies such as IBM, NOKIA, Microsoft, Yahoo, Google, are using agile methods for producing software (Chung and Drummond, 2009; Cohan and Glazer, 2009; Fulgham et al., 2011; Laanti et al., 2011); simultaneously large numbers of managers are interested in implementing agile methods in their companies. However, we did not find any serious report about failing on replacing traditional SD with Information Technology, University Putra Malaysia (UPM), 43400, Serdang, Malaysia 
Taghi Javdani Gandomani et al. / Journal of Computer Science 9 (5): 620-625, 2013

agile methods, but, this mutation in producing software is not simple and quick and should be certainly done after removing the probabilistic barriers and problems. Since the change of the process affects all aspects of a company such as technical, management, personal and cultural aspects, encountering with barriers and hindrances is quite inevitable. Lack of the knowledge about these challenges makes agile transformation too hard, even in small and medium companies.

Following sections in this study subsequently explain agility, traditional versus agile SD methods, challenges of migrating to agile methods and finally summary and conclusion.

\section{AGILITY}

Agile software development emphasize on agility in software production. Agility in software production is based on some defined values. Agile manifesto emphasizes on four particular values (Beck et al., 2001):

- Individual and interaction over processes and tools

- Working software over comprehensive documentation

- Customer collaboration over contract negotiation

- Responding to changes over following plan

Iteration and incremental development, quick delivery, continuous quality improvement, individual oriented process, self-organizing teams, embracing requirement change (even in last stages), simplicity and particular pay attention to customer satisfaction are principles of agile SD methods (Singh and Soni, 2011; Williams, 2012). Obviously, achieving some or all of them requires fundamental changes in traditional SD methods. It should be said that some of the agile methods mainly focus on software development, like XP (Chromatic, 2013) and some others emphasize on project management, like Scrum (Rubin, 2012). Indeed agility affects on both software development and software project management.

\section{TRADITIONAL VERSUS AGILE}

Emergent of agile methods was a reaction to traditional methods. Investigating on traditional and agile SD methods indicates a distinct and deep gap between them. Differences between them are in all aspects of their concepts and activities. Table 1 compares these two approaches briefly (Conboy et al., 2011). In a deeper view, the differences provided in the above table could be categorized as bellow.
Table 1. Comparing traditional and agile SD methods

\begin{tabular}{|c|c|c|}
\hline Properties & Traditional SD & Agile SD \\
\hline Attitude & Predictive & Adaptive \\
\hline Project size & Large & Small \\
\hline Team size/mindset & Large/disciplined & Small/innovative \\
\hline $\begin{array}{l}\text { Project management } \\
\text { model }\end{array}$ & Autocratic & Decentralized \\
\hline $\begin{array}{l}\text { Change attitude } \\
\text { changes }\end{array}$ & Resistant against & $\begin{array}{l}\text { Embracing } \\
\text { changes }\end{array}$ \\
\hline Documentation & Comprehensive & Light and abstract \\
\hline Upfront planning & Comprehensive & Limited \\
\hline Life cycle & Tied and bound & $\begin{array}{l}\text { Unlimited } \\
\text { iteration }\end{array}$ \\
\hline $\begin{array}{l}\text { Organizational } \\
\text { culture }\end{array}$ & $\begin{array}{l}\text { Command and } \\
\text { control }\end{array}$ & $\begin{array}{l}\text { Leadership and } \\
\text { collaboration }\end{array}$ \\
\hline $\begin{array}{l}\text { Return of } \\
\text { Investment }\end{array}$ & $\begin{array}{l}\text { At the end of } \\
\text { the project }\end{array}$ & Early stages \\
\hline
\end{tabular}

\subsection{Management}

From management point of view, agile methods are based on the leadership and personnel innovation. This means that agile methods emphasize on collaboration in projects. Despite of agile methods, in traditional methods, top and middle level managers play "command and control" role and personnel should obey their commands (Ghanam et al., 2012; Pikkarainen et al., 2012; Yang et al., 2009a). Both approaches by acceptance of their own disadvantages emphasize on their positive outcomes and advantages. Finally, decentralized management in agile is against of the centralized and autocratic management in traditional methods.

\subsection{Organization}

While in traditional software development methods organization is completely defined based on the organizational foundations, there is no intensive idea about organization and organization plan in agile methods. People in traditional methods have pre-defined and strict role and are controlled directly, but in agile methods teams are self-organized and individualoriented (Hoda et al., 2011). In these methods individual creativity could be seen in the best way.

\subsection{Project}

In traditional approach, projects are usually large and manage by large size teams. In these methods upfront comprehensive planning, scheduling and budgeting are essential. Despite of traditional methods, in agile methods, projects are mostly small and medium and are managed without comprehensive upfront planning. If necessary, sometimes a limited but not intransitive upfront planning could be performed (Shi et al., 2011). 


\section{CHALLENGES IN AGILE TRANSFORMATION}

Most of the companies interested in agile are those which have many years of experience in traditional methodologies. For moving to agile methods, they should confront with barriers and hindrances (Srinivasan and Lundqvist, 2010). The roots of most of the barriers are organizational culture and structure of which is necessary for traditional approach.

Generally, the barriers of moving to agile could be summarized in next sections.

\subsection{Organization and Management Related Challenges}

Social structure of organizations is influenced by organizational culture (Kautz et al., 2009). Generally in companies, organizational culture exerts significant influence on innovative practices, social negotiations, problem solving strategies, decision-making processes and planning and control mechanism (Pikkarainen et al., 2012). Clearly, changing mindset of people and their organizational culture could not be an easy process (Ghanam et al., 2012). To transforming from traditional to agile methods, management style should be changed from "command and control" to "leadership and collaboration" (Yang et al., 2009b). It could be facilitated by right blend of cooperation and autonomy. This approach causes enough flexibility and responsiveness in organization and provides advantages of synergy simultaneously. The role of project manager should be altered from planner and controller to director and coordinator (Moe et al., 2009; Monteiro et al., 2011). In fact he/she should coordinate the collaborative efforts of team members; meanwhile, he/she should ensure that creative ideas are reflected in final decision. Group decision making is also an issue, especially in allocation of development resources, alignments of strategic product line and performing development and maintenance tasks in teams (Moe et al., 2012). Another issue is that sometimes project managers could not ignore their previously authority and role. Changing mind set of project managers take a long time and need enough mentoring (Pikkarainen et al., 2012).

In this subject, another challenge is documentation; while in traditional methods knowledge management is based on heavyweight documentation, in agile methods, documentation is limited and knowledge is mostly tacit and reside in the head of the development team members
(Levy and Hazzan, 2009) of course, this approach changes power balance in organization from managers to individuals and could be a big issue mainly for managers that are aware about people and have negative mindset and experiences in human resource in their working life. This challenge could be decreased by defining appropriate knowledge management strategy and distribution of knowledge in different level of organization. This is a fertilize area for doing research in both academic and industrial environment.

There also many issues in regarding to the distributed development organizations. The biggest issue is communication. In such companies due to the distance, face to face meeting is difficult, also time zone offset makes communication harder (Kamaruddin et al., 2012; Lee and Yong, 2010). Cultural difference is also reported as a critical challenge in multi international sites (Dorairaj et al., 2012; Iivari and Iivari, 2011).

\subsection{People Related Challenges}

Achieving to a cooperative process based on the communication and collaboration between members who value and trust each other is critical for success of agile methods (Dorairaj et al., 2012; Offner et al., 2011). Human aspects most of the time acts as an obstacle in agile adoption (Tolfo et al., 2011). In some of the agile methods there are some individual centered activities e.g. Pair programming in XP (Chromatic, 2013). In this case, managers should select appropriate personnel and provide them necessary training, mentoring and creating a set of work practices that promote process excellence (Srinivasan and Lundqvist, 2010). These types of activities especially for senior traditional developers could not be implemented easily. Another challenge is related to customers. In agile methods customer is one of the development team members and all decision is based on different attitudes, goals and experiences. In this case, some traditional project managers could not adapt themselves with new situation. Customers play a critical role in success of agile methods and they should be responsive, collaborative, authorized, committed and knowledgeable (Conboy et al., 2011). Having such customers is not easy and this role could be as a barrier in success of agile projects especially when they join the team for the first time. About coaching process in movement to agile because of being a lot of human factors affected the process; coaches need to be patient (Srinivasan and Lundqvist, 2010). Managers should pay enough attention to assign an experienced and professional coach in their teams. To sum up, lack of enough training, coaching and mentoring is a critical issue in this area. 


\subsection{Process Related Challenges}

Changing attitudes and moving to agile activities from rigid, adequate and planned activities is not available without spending enough time, effort and investment. In fact, altering attitudes and activities is problematic, especially for companies with high level of CMMI (Babuscio, 2009).

In traditional methods processes are based on defined/standard activities and measurement while processes in agile methods are based on uncertain activities that support rapid development and high quality production (Singh and Soni, 2011). In agile methods finding appropriate measurement practices is a debatable issue. Despite of traditional methods, there are a few popular and acceptable measurement practices in agile methods (Javdani et al., 2012). So, sometimes expectation of traditional developers to finding adequate and documented measuring tools in agile methods causes confusing.

Changing process model from traditional life cycle model to agile (evolutionary and iterative) is an obstacle in altering approaches; because this change has significant influence on strategies, tools, role of the people and techniques. Implementing some agile activities such as continuous integration, developing upfront test code and frequent testing in traditional software developers is hard.

Another issue is choosing appropriate agile method. However all of them are based on agile values, but there are many different practices and activities in them. They are different in priorities, implementation, project and team size, iteration time, code ownership and other factors. Unfortunately there is no unified agile approach, so, organizations should decide about their appropriate agile method. Wrong selection of appropriate method, cause more efforts and cost and even may cause failure in agile movement.

\subsection{Technology and Tools Related Challenges}

However technological issues in migrating to agile are less than other issues, but companies should be aware of them. Using non-flexible tools and hardware is a barrier in moving to agile. Companies should use tools that can supply incremental evolution, continuous integration, re-working, version management and other agile technologies. Thus considering enough investment and training, could help organization for confronting these challenges. Tools especially in multi sites organization is a big challenge (Brockmann and Thaumuller, 2009). Providing appropriate tools for doing practices in the best way is necessary to using agile methods in distributed development environment.

\section{CONCLUSION}

While benefits and advantages of development of agile methods in software companies encourage them to use these methods, they should consider challenges and barriers in moving process. Since values in agile methods are completely different from traditional methods, activities, practices and roles are also different. Thus, moving to agile affects all aspects of organizations. All members of organization should be ready to confront with a lot of challenges in transforming process. The challenges are mainly in organizational culture, management, people and process area. The main origins of these challenges are organizational culture and structure. Also, relinquishing a process centric model and moving to people centric one is not simple. Managers should be cautious about when and how they could change their producing methods. Enough time, effort and training could help them for this strategic decision.

\section{ACKNOWLEDGMENT}

This study was supported by the International Graduate Research Fellowship (IGRF) by University Putra Malaysia (UPM).

\section{REFERENCES}

Babuscio, J., 2009. How the FBI learned to catch bad guys one iteration at a time. Proceedings of the Agile Conference, Aug. 24-28, Chicago, IL, pp: 96100. DOI: 10.1109/AGILE.2009.52

Beck, K., M. Beedle, A.V. Bennekum, A. Cockburn and W. Cunningham et al., 2001. The Agile Manifesto. Software Development.

Boehm, B., 2002. Get ready for agile methods, with care. Computer, 35: 64-69. DOI: 10.1109/2.976920

Brockmann, P.S. and T. Thaumuller, 2009. Cultural aspects of global requirements engineering: An empirical Chinese-German case study. Proceedings of the 4th IEEE International Conference on Global Software Engineering, Jul. 13-16, IEEE Xplore Press, Limerick, pp: 353-357. DOI: 10.1109/ICGSE.2009.55

Chromatic, 2013. Extreme Programming Pocket Guide. 1st Edn., O'Reilly Media, Sebastopol, CA., ISBN10: 1449366643, pp: 108. 
Chung, M.W. and B. Drummond, 2009. Agile at Yahoo! From the Trenches. Proceedings of the Agile Conference, Aug. 24-28, IEEE Xplore Press, Chicago, IL, pp: 113-118. DOI: 10.1109/AGILE.2009.41

Cohan, S. and H. Glazer, 2009. An agile development team's quest for CMMI® maturity level 5 . Proceedings of the Agile Conference, Aug. 24-28, IEEE Xplore Press, Chicago, IL, pp: 201-206. DOI: 10.1109/AGILE.2009.24

Cohn, M., 2009. Succeeding with Agile: Software Development Using Scrum. 1st Edn., Pearson Education, ISBN-10: 0321660560, pp: 504.

Conboy, K., S. Coyle, X. Wang and M. Pikkarainen, 2011. People over process: Key challenges in agile development. IEEE Software, 28: 48-57. DOI: 10.1109/MS.2010.132

Dingsoyr, T., S. Nerur, V. Balijepally and N.B. Moe, 2012. A decade of agile methodologies: Towards explaining agile software development. J. Syst. Software, 85: 1213-1221. DOI: 10.1016/j.jss.2012.02.033

Dorairaj, S., J. Noble and P. Malik, 2012. Understanding lack of trust in distributed agile teams: A grounded theory study. Proceedings of the 16th International Conference on Evaluation and Assessment in Software Engineering, (EASE' 12), Ciudad Real, pp: 1-20.

Fulgham, C., J. Johnson, M. Crandall, L. Jackson and N. Burrows, 2011. The FBI gets agile. IT Professional, 13: 57-59. DOI: 10.1109/MITP.2011.88

Ghanam, Y., F. Maurer and P. Abrahamsson, 2012. Making the leap to a software platform strategy: Issues and challenges. Inform. Software Technol., 54: 968-984. DOI: 10.1016/j.infsof.2012.03.005

Glazer, H., 2010. Love and marriage: CMMI and agile need each other. CrossTalk, 23: 29-34.

Hoda, R., J. Noble and S. Marshall, 2011. Developing a grounded theory to explain the practices of selforganizing Agile teams. Empirical Software Eng., 17: 609-639. DOI: 10.1007/s10664-011-9161-0

Iivari, J. and N. Iivari, 2011. The relationship between organizational culture and the deployment of agile methods. Inform. Software Technol., 53: 509-520. DOI: $10.1016 /$ j.infsof.2010.10.008

Javdani, T., H. Zulzalil, A.A.A. Ghani and A.M. Sultan, 2012. On the current measurement practices in agile software development. Int. J. Comput. Sci., 9: 127-133.

Kamaruddin, N.K., N.H. Arshad and A. Mohamed, 2012. Chaos issues on communication in Agile Global Software Development. Proceedings of the Business Engineering and Industrial Applications Colloquium, Apr. 7-8, IEEE Xplore Press, Kuala Lumpur, $\quad$ pp: 394-398. DOI: 10.1109/BEIAC.2012.6226091
Kautz, K., C.F. Pedersen and O. Monrad, 2009. Cultures of Agility-Agile Software Development in Practice. AIS Electronic Library (AISeL).

Laanti, M., O. Salo and P. Abrahamsson, 2011. Agile methods rapidly replacing traditional methods at Nokia: A survey of opinions on agile transformation. Inform. Software Technol., 53: 276290. DOI: $10.1016 /$ j.infsof.2010.11.010

Lee, S. and H.S. Yong, 2010. Distributed agile: Project management in a global environment. Empirical Software Eng., 15: 204-217. DOI: 10.1007/s10664009-9119-7

Levy, M. and O. Hazzan, 2009. Knowledge management in practice: The case of agile software development. Proceedings of the ICSE Workshop on Cooperative and Human Aspects on Software Engineering, May 17-17, IEEE Xplore Press, Vancouver, BC., pp: 6065. DOI: 10.1109/CHASE.2009.5071412

Moe, N.B., A. Aurum and T. Dyba, 2012. Challenges of shared decision-making: A multiple case study of agile software development. Inform. Software Technol., 54: 853-865. DOI: 10.1016/j.infsof.2011.11.006

Moe, N.B., T. Dingsoyr and K. Oyvind, 2009. Understanding shared leadership in agile development: A case study. Proceedings of the 42nd Hawaii International Conference on System Sciences, Jan. 5-8, IEEE Xplore Press, Waikoloa, HI, pp: 1-10. DOI: 10.1109/HICSS.2009.480

Monteiro, C.V.F., F.Q.B.D. Silva, I.R.M.D. Santos, F. Farias and E.S.F. Cardozo et al., 2011. A qualitative study of the determinants of self-managing team effectiveness in a scrum team. Proceedings of the 4th International Workshop on Cooperative and Human Aspects of Software Engineering, (SE' 11), ACM Press, New York, USA., pp: 16-23. DOI: 10.1145/1984642.1984646

Offner, A., S. Swindler, G. Padula, A. King and J. Fedora, 2011. Change management: Developing a tool to foster adaptive collaboration. Proceedings of the International Conference on Collaboration Technologies and Systems, May 23-27, IEEE Xplore Press, Philadelphia, PA., pp: 606-611. DOI: 10.1109/CTS.2011.5928743

Pikkarainen, M., O. Salo, R. Kuusela and P. Abrahamsson, 2012. Strengths and barriers behind the successful agile deployment-insights from the three software intensive companies in Finland. Empirical Software Eng., 17: 675-702. DOI: 10.1007/s10664-011-9185-5 
Rubin, K.S., 2012. Essential Scrum: A Practical Guide to the Most Popular Agile Process. 1st Edn., AddisonWesley Professional, Michigan, ISBN-10: 0137043295, pp: 504.

Shi, Z., L. Chen and T.E. Chen, 2011. Agile planning and development methods. Proceedings of the 3rd International Conference on Computer Research and Development, Mar. 11-13, IEEE Xplore Press, Shanghai, pp: 488-491. DOI: 10.1109/ICCRD.2011.5764064

Sims, C. and H.L. Johnson, 2012. Scrum: A Breathtakingly Brief and Agile Introduction. 1st Edn., Dymaxicon, San Francisco, CA., ISBN-10: 193796504X, pp: 54.

Singh, N.P. and R. Soni, 2011. Agile software: Ensuring quality assurance and processes. Proceeding of the International Conference on High Performance Architecture and Grid Computing, Jul. 19-20, Chandigarh, India, pp: 640-648. DOI: 10.1007/9783-642-22577-2_86
Srinivasan, J. and K. Lundqvist, 2010. Agile in India: Challenges and lessons learned. Proceedings of the 3rd India Software Engineering Conference, Feb. 25-27, ACM Press, New York, USA., pp: 125-130. DOI: $10.1145 / 1730874.1730898$

Tolfo, C., R.S. Wazlawick, M.G.G. Ferreira and F.A. Forcellini, 2011. Agile methods and organizational culture: Reflections about cultural levels. J. Software Maintenance Evolut., 23: 423-441. DOI: $10.1002 / \mathrm{smr} .483$

Williams, L., 2012. What agile teams think of agile principles. Commun. ACM, 55: 71-76. DOI: $10.1145 / 2133806.2133823$

Yang, C., J. Liu, H. Chen and X. Luo, 2009a. Adaptive optimization of agile organization of command and control resource. J. Syst. Eng. Elect.., 20: 558-564.

Yang, H., S. Huff and D. Strode, 2009b. Leadership in software development: Comparing perceptions of agile and traditional project managers. Proceedings of the 15th Americas Conference on Information Systems, Aug. 6-9, San Francisco, California, USA., pp: 184-184. 